Journal of Teacher Education for Sustainability, vol. 13, no. 1, pp. 19-28, 2011

\title{
OPEN VERSUS CLOSED FORMS OF KNOWLEDGE ASSESSMENT IN A BLENDED LEARNING ECOSYSTEM
}

\author{
Peter Purg \\ Institute and Academy of Multimedia, Slovenia
}

\begin{abstract}
The article investigates whether two opposite assessment methods, open wiki (online textand-media collaboration) and closed multiple-choice test, can together assure a balanced addressing of the learner both as an information processor and a social entity. Comparing students' experience and satisfaction with collective knowledge construction in a moderated wiki on the one hand and knowledge testing in an online multiple choice test on the other hand, the research differentiates full-time and part-time students of a blended course. This is presented upon broader data on comparable students' populations who were assessed with open-ended oral exam questions, regular homework tasks and seminar work papers. An updated notion of media ecology conceives knowledge testing and instructional feedback as parts of an informational ecosystem: only a coherent yet internally differentiated learning environment can accurately define significant educational trends and recommendations - such that question assessment methods and signpost the development of education towards sustainability.
\end{abstract}

Key words: assessment, wiki, test, blended, media ecology

\section{Learning as information processing}

The information society of the recent century has been progressing towards the so-called knowledge society for more than a decade now, which means that the (formative) competence of information linking and application should have surpassed mere (informative) ownership of data. It seems as if the immanent dependence of knowledge construction and management on information and communication technology (ICT) is becoming stronger ever since, even more so from the perspective of sustainable development. The rapid socialeconomic changes in the contemporary society indeed call for flexibility of knowledge and skills not only among the workforce, the citizens, the students, but first and foremost among teachers and trainers as primary media of education. So-called new media, such as the internet (especially in its recent mobile developments towards omnipresence), address the human natural information processing potential - that has albeit ever since been multi- 
channel, parallel and complex (Giesecke, 2002). So-called multimedia is innately pertaining to the human body and concrete space experience, and, as such, it is being increasingly emphasized in all educational areas (Mishra \& Sharma, 2004). On the other hand, computers especially with their current global networking, potential and accessibility of the so called cloud - can not only s(t)imulate natural but also facilitate technical information processing, enabling both improved learning performance (as knowledge quality) and accessibility (as quantifiable knowledge availability). The reflection of this (multi)media dimension has been insufficiently coded into memorandums on lifelong learning and digital literacy, as both the man- as well as the machine-centred ICT development agendas seem to lack this important aspect.

ICT is indeed closely connected to the globalization process playing an important role in renewing educational processes. That, in turn, significantly correlates with social and cultural change. Sustainable development, thus, needs to influence all areas of human existence by connecting and balancing the many possibilities opened up by new technologies. This becomes even more apparent after the United Nations (UNECE, 2009) and the European Union (EC, 2008) declaratively accepted sustainable development as a priority paradigm in implementing the important strategies of cultural, social and economic development. The Guidelines and Recommendations for Reorienting Teacher Education to Address Sustainability promote the use of ICT to help students take responsibility for finding information and evaluating it as well as ultimately taking responsibility for aspects of their own learning (UNESCO, 2005). Yet, these visions and strategies so far fail to recognize the sustainability potential of e-learning not only as a mere vehicle for promoting values and contents of sustainable development, but also as a method that can immediately implement such guidelines. The great potential of ICT to support knowledge management (Mansell \& Wehn, 1998) is critically neglected, as there has been little understanding of how new technologies in education can unburden and motivate the learner and promote communication and collaboration among people and cultures. Educators can link natural human communication and collaboration potential with multimedia interaction possibilities of the new media in so-called blended learning settings (combining online and presence teaching phases). Together these methods can contribute to realizing the vision of a sustainable society, both in the field of formal and non-formal education.

\section{Media ecology updated}

During the historical development of ICT, the modes of communication were gradually restricted to (i.e. made dependent of) machine-processed or -mediated signs and signals. But soon, though the ICT boom was still in full swing, the technology was being pragmatically optimized, the human body as the primary communication medium was revoked and revaluated. This was first done by the way of counter-dependency, followed by balanced autonomy (Giesecke, 2002) integrating face-to-face, mediated, sign-based and multimodal communication. Currently, information-to-knowledge society may be facing quite a similar balancing process in the realm of education: the e-learning euphoria has hardly begun ebbing, yet there are already sound considerations of various forms of face-to-face communica- 
tion compensating for the lack of motivation as an inherent problem of technologymediated learning (Purg, 2006). This can be countered by a balanced use of social and other digital (multi)media (Purg \& Zakrajšek, 2009) that, however, should be used consequently and in a differentiated way. Such use shall be highlighted in this paper, providing empirical data on the comparison of closed and open forms of knowledge construction and assessment.

In order to consistently contextualize the dichotomy of open versus closed information processing, there is an evident need for a new conceptual framework that would allow a balanced implementation and representation of (and sensitization for) all informationexchange types. The author believes that these are dealt with particularly convincingly by media ecology as a discipline initially defined by Postman (1970) to look "into the matter of how media of communication affect human perception, understanding, feeling, and value; and how our interaction with media facilitates or impedes our chances of survival" ( $p$. 161). Nystrom (1973) defined the concept of media ecology with a viable reference to information processing within complex communication systems as environments. Sandbothe (2004), however, offered a more contemporary understanding of media ecology as the one of counterbalance, re-establishing the "neglected" media. In the nineties, this may have been ICT - computer and the internet, yet nowadays there is an evident regain of body media and the institutionalized media of verbal and non-verbal scientific communication (Sandbothe, 2004). This could readily explain the mainstreaming of blended learning models, where face-to-face teaching and learning is closely intertwined with online communication and the use of the web or mobile media. Many recent cultural developments and phenomena show that forms of collective intelligence, as enabled technically through the internet, are deeply rooted in the bodily experience of the human self - and of the other. These dimensions of (the so called emotional) intelligence have been neglected by educational and training systems far too long and were surely missing in the reason-dominated elearning conceptions of the recent two decades. Indeed, according to Sandbothe (2004), there is an evident need for a "synaesthetic" revalidation of these dimensions under the new conditions of the media society.

A viable paradigm for a well balanced usage of technical and physical media (as forms of communication) may be offered by Giesecke (2002) in his vision of media ecology that qualifies no leading medium as the "key" (Ger. "Schluesselmedium”) that could assure perfect knowledge and/or skill acquisition, but instead a well-balanced structure of different information systems. It is important to note that the concept of an information system in this context may be scoped at the level of culture as common, collective practice and on the level of both a singular human being and a group of people, for instance, learners or trainees. An integration of technical, psychological, biogene and social information systems is mandatory in order to defy the monopoly of one sole processing type: as it was the case for written communication in the past half millennium, the contemporary situation shows clear signs of ICT (computers, mobile phones, tablets, etc.) gradually taking the leading role and gaining the grounds of "the book" as the declining "monomedium". Moreover, face-to-face communication is gradually coming into the position of counterbalance against the aforementioned two, offering a fair chance of (polycentric) media balance across cultures - note 
here that multimedia technologies have always been seeking to challenge the complexity of present face-to-face communication, unbound by the limits of space and time.

The so-called new media are, according to Sandbothe (2004) and Giesecke (2002), to be considered as only one further barycentre in the newly balanced media-scape. After an initial euphoric and overvaluing of ICT and multimedia, a sober coexistence is to be sought historically with other paradigms of information processing. So the new media - every time they emerge as new - not only enable original ways of structuring and distributing knowledge, they also offer new contexts for re-evaluation and rediscovery of old media, such as writing by hand or face-to-face communication. At the present point of equally held stakes and flexible positioning of (meta)perspective, instead of the mono-causal information processing in terms of "either or" - a fair chance for an inclusive "as well as" paradigm is evident. In the realm of teaching and training, this would mean that, by keeping up and reflecting upon the differences, all channels or modes of communication should be carefully balanced. Practically, this would probably call for carefully investigating the prevalent modes and adjusting the portions of differently mediated (or indeed assessed) learning materials to the topic, the pedagogical aim and the (cultural, disciplinary, generational, etc.) structure of the trained/taught group (Purg, 2006). Still, this paper focuses on text-based knowledge construction and assessment, since it is presumed that the comparison of their radical closed (multiple-choice test) and open (wiki) forms presents an evident-enough instance of different information exchange types.

\section{Learning together in an ecosystem}

A wider ecological perspective also offers several other relevant considerations that cannot be dealt with in the scope of the present paper, but ought to be considered for further investigations of this kind. Their relevance for the present topic may be drafted through themes such as mutual dependency of beings (as, for instance, information entities), dynamic equilibrium of systems and the postulate of resource scarcity - both well-applicable to information processing in the context of ICT. It is thus, even in a stricter context of educational informatics, quite obvious that "the educational domain is an excellent place to explore the ecological approach, since there is every chance of a very high bandwidth of interaction between the learner and the system" (McCalla, 2004, p. 6). According to McCalla (2004), such an approach could, in a rather pragmatic way, underlie the designs of, among others: a study aid, a recommender system (content relevant to learner's current task), an instructional planner (individualized curriculum of study), a group formation tool (grouping learners according to tasks or subjects), a peer-help seeker, a reminder system (for instance, RSS feed, the "Real Simple Syndication" of website updates to clients, mobile devices etc.), a learner-type-sensitive evaluation tool (for instance, for instructional and cognitive scientists), an end-use (automated, learner-type-adjusted) tagging system, an "intelligent garbage collection system" (reviewing relevance of educational contents and methods) etc. (ibid.)

Tilbury (2007) defines several key competencies of education for sustainable development (ESD) that support the implementation of ICT for sustainability: complex systems in the context of educational settings demand a system(at)ic approach to communication 
that should incorporate both human and technological factors. Peled, Peled and Gad (1994) understand classrooms and schools as culturally dependent systems within a "culturalecological framework" (p. 58) that enables a definition of several indicators for the identification of ecological change processes (in classroom or school): cultural-ecological change generates educational innovations (ibid.), although it needs to be paralleled by "treatmentspecific types" (p. 58); interactions with information technology (IT) should be "extensive and varied" (p. 58), but IT must be "mindfully integrated" into the (historically) printdominated classrooms. Here, interestingly, the print-oriented situation of 1994 still holds a comparative potential to the continuous book-and-paper heavy teaching practices of 2011 worldwide and across cultures. In such a case, the use of multiple perspectives proves crucial not only in terms of online versus offline knowledge construction, but also in different combinations within the multimedia model (picture-text, sound-picture, etc.), which relates to a heterogeneity of knowledge construction and knowledge assessment practices highlighted in this paper. Alternating learning environments should be understood as awarding "unique contributions" (ibid.) calling for continuous and "mindful" (ibid.) change of course designs and learning methodologies.

The 21 st century is seeing important developments in the use of technologies for social networking - not only in real life (for instance, Facebook) and career (for instance, Linke$\mathrm{dIn}$ ) or marketing and citizenship (for instance, Twitter), but also in the realm of e-learning. All the major course management applications, such as Moodle or Blackboard, have long started introducing aspects of web 2.0 into educational ICT, ranging from individual elements, such as wiki, blog or instant messaging, to systemic solutions, such as hubfunctionality, fostering learning in trans-local settings and intercultural community-building in educational contexts. Results in a recent research of Lambert, Kalyuga and Capan (2009) suggest that prior experience in distance education and technology is associated with lower anxiety and higher engagement, which agrees with the present author's own preliminary research (Purg \& Zakrajšek, 2009) showing that web 2.0 technologies do not impose excessive levels of mental load, if proper curricular design and implementation are secured. It is obviously important that intrinsic and extraneous cognitive loads are reduced sufficiently by providing an organized and clear course design and selecting engaging materials and activities suitable for different levels of learner experience and expertise (Lambert et al., 2009).

\section{Formative versus informative - Wikis versus tests}

In this study, the aforementioned assumptions and predictions are checked against a representative body of quantitative empirical data from a topical questionnaire and complemented with qualitative observations. The research has been carried out for two years on several comparable groups of students attending the same course "Introduction to Media" as the introductory course of a recently Bologna-renewed higher vocational education programme "Media Production" in Slovenia. The course was conducted under the leadership of the same teacher (author of this paper), with exactly the same contents, aims, outcomes and also learning, teaching and assessment strategies - yet conducted at three different 
schools. As a rule, full-time students attend courses in the morning or at midday, whereas part-time students attend courses in the afternoon and mostly combine studying with regular work. All course runs lasted 8-10 weeks in the winter semester, beginning in October and completing in mid-December. Moodle learning management system for blended learning (no paper materials were transacted whatsoever) was adopted. Table 1 shows the comparison and calculation of "hours per ECTS credit point" in order to illustrate the workload differences (Purg \& Zakrajšek, 2009), as assessed by the students themselves.

Table 1. Workload questionnaire response overall statistics in the academic year 2008/2009 and the academic year 2009/2010

\begin{tabular}{lllll}
\hline Year of study & \multicolumn{2}{c}{$\mathbf{2 0 0 8 / 2 0 0 9}$} & \multicolumn{2}{c}{$\mathbf{2 0 0 9 / 2 0 1 0}$} \\
\cline { 2 - 5 } Mode of study & Full-time & Part-time & Full-time & Part-time \\
\hline Group size (students in group) & $36+38$ & 22 & $40+42$ & $42+20+22$ \\
\hline Hours of work per ECTS credit & 24 & 20 & 32 & 24 \\
\hline
\end{tabular}

The group sizes of all runs were comparable (the smallest group had 20 and the largest had 42 students), a total of 96 students were included in the academic year 2008/2009 run, and 166 students were included altogether in the academic year 2009/2010. The questionnaire response was always between 50 and $80 \%$ per group. Apart from typical workload-focused questions, the students were also asked to assess their general satisfaction with specific elements of e-learning used in this blended course (10 face-to-face meetings combined with intensive online work). The results of selected, topic-relevant elements related to knowledge assessment are compared in Figure 1, presenting the average response by each type of students within an academic year.

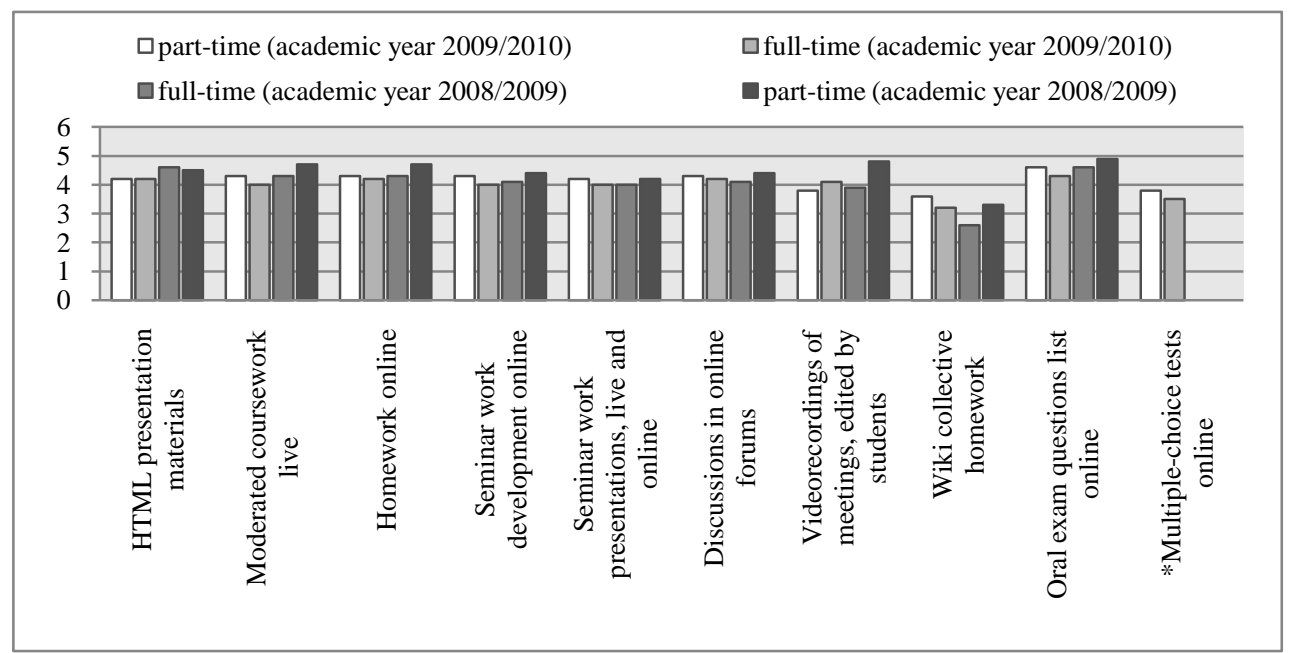

*for multiple-choice test online, there is no data for the academic year 2008/2009

1 - completely unsatisfied; 5 - completely satisfied

Figure 1. Students' satisfaction with specific e-learning elements 
It is possible to conclude that all other forms of assessment and activities were preferred better than the wiki collective homework assignment (introduced only in the academic year 2009/2010). In this assignment, students had to, within a course-internal wiki, collectively construct answers to subject-related questions that (in the form of a website) contained text, pictures, links (also to video content), etc. Another form of assessment, short (online, timelimited) multiple-choice tests was introduced besides the methods used so far (regular homework submission online, seminar work development and submission online, seminar work presentations live and through online video, final oral exam). Interestingly, this most restrictive and stressing assessment element scored only slightly below all others, yet was surprisingly preferred by all surveyed groups over the wiki, whereby wiki even had a considerably lower (cumulative) final-mark impact than the tests. Part-time students generally show higher appreciation levels of e-learning elements than full-time students - which could be due to their increased absence from live meetings because of work- and familyrelated obligations that they can compensate for by studying online. Whereas, surprisingly, a significant increase of study stress can be indicated with full-time students, who in the academic year 2009/2010 obviously show greater dissatisfaction with the further step in assessment methods differentiation - tests and wikis were the only newly introduced assessment element as compared to the previous year's (the academic year 2008/2009) course design. This could also be the main reason for the increase in average hour-per-credit value of the course (Table 1), showing that students had to work more hours per credit than before.

In Figure, 2 it is possible to see that multiple-choice tests were agreed by the students to be the most exact indicator of their knowledge.

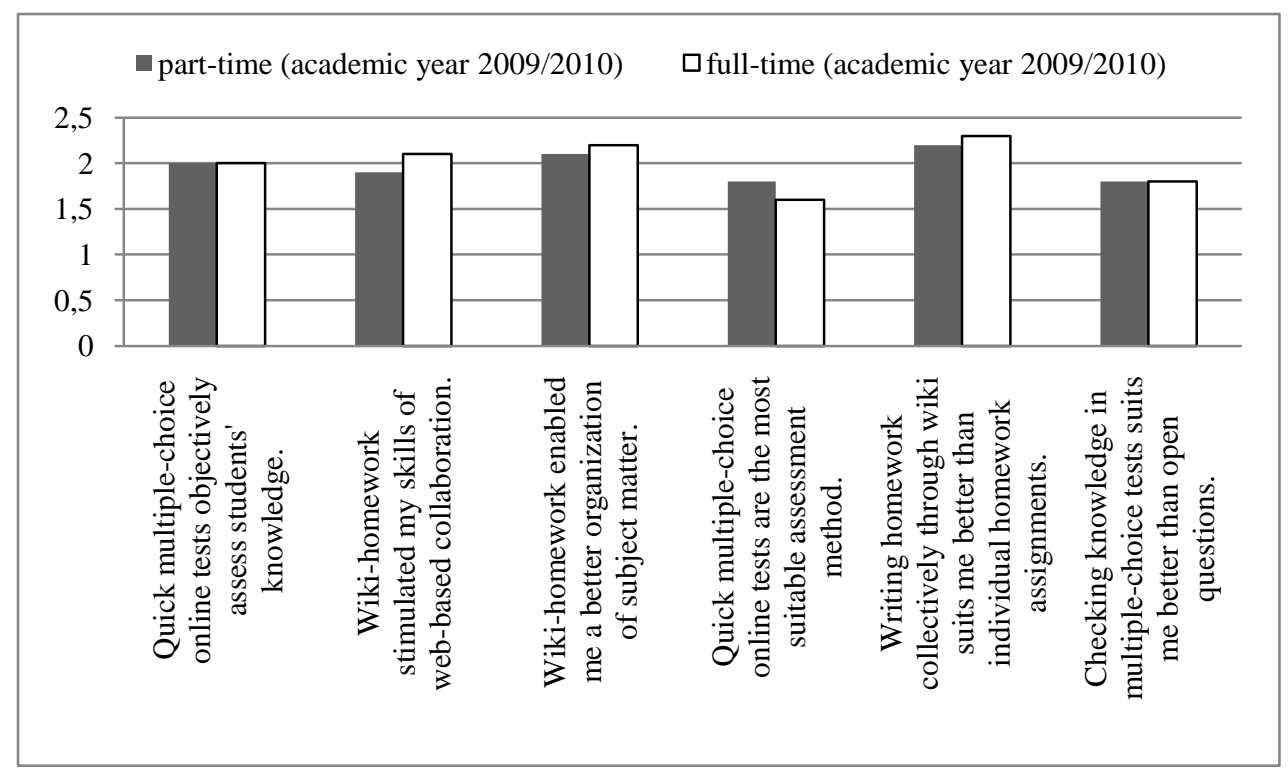

$1-$ true; $2-$ partly true; $3-$ not true

Figure 2. Students' opinion on assessment relevance of wikis and tests 
Thus, they were unanimous in the opinion that quick multiple-choice online tests are the most suitable assessment method, even if they often reported high levels of stress due to a strict computer-controlled time limit (5 minutes for 8 randomly chosen questions). Checking knowledge in a time-limited multiple-choice form obviously suited students much better than open questions (final oral exam). Whereas collaborating on the homework task collectively through wiki suited them far less than individual homework assignments submitted into a drop-box. It should be noted that each collective homework wiki assignment was topically linked to the respective individual homework assignment, submitted in a document file. Still, the wiki-homework enabled most of the students a better organization of subject matter, thus after all proving to be of positive impact to knowledge construction, even if not enjoyed as an assessment form. It needs to be added here that - for experimental reasons - the wiki process was not explicitly followed or participated in by the teacher, instead, only short oral feedback was given to students after each session as to stimulate a more spontaneous and equal participation. A next possible step of course design development could, thus, be towards participatory assessment forms, since Knipe and Edwards (2009) prove that, by using a wiki to create exam questions, a web-2.0 version of the traditional open-book exam is possible, bringing about credible assessment results and raising motivation - if conducted correctly and carefully. Quite similar, as in the example provided, this could only be possible if careful moderating of the collective wiki usage is provided interactively by the teaching authority.

\section{Blended classrooms for sustainability}

As a typical and up-to-date form of formative assessment, wikis, much like other web 2.0 elements in education (Greenhow, Robelia, \& Hughes, 2009), call for mindful implementation. This is because, by enabling quick and simple access - and by false analogy to noneducational uses in social networking or other collaborative online processes - new technologies can easily mislead the student into unpremeditated construction of text or mediarich contents. Then again, they may seem just a misused assessment form, or even pose a threat to students' individuality, identity or privacy. Such unbearable lightness of web participation as in a collective wiki assessment can obviously be positively counterbalanced by rigid informative assessment, such as through individual closed tests. Different types of information processing can thus be - and especially with(in) new technologies - sustained together in a type-different yet internally balanced informational ecosystem. Such a claim can be further supported by the fact that the aforementioned course has achieved high students' satisfaction scores, even though it was heavily laden with online, live activities and manifold forms of assessment. It also showed particularly high and increasing learning motivation indicators - even in the face of a comparatively immense self-assessed workload.

In terms of media ecology, the aforementioned arguments plead for a careful and well balanced use of resources, combining different and complementary ways of information processing for a clearly defined learning objective (Purg, 2006). Emerging computer technologies increasingly implemented in the realm of education, such as cloud computing or social operating systems, are, according to Greenhow et al. (2009), likely to intensify the 
participatory and creative practices in both virtual and physical classrooms. The author finds that dependence on data and information - arguably here also within assessment processes - shall be equal to or even replaced by an emphasis on creating, developing, and sustaining human relationships. New media in education can foster interactive knowledge acquisition that is more sustainable in terms of being focused onto the learner's actual needs, information-processing preferences and social (network) capacities. And, indeed, only if a learning environment is coherent in terms of social structure and informationprocessing and also internally differentiated, can it allow for accurate revelations of significant educational trends and practical recommendations - such that question and signpost the development of education towards sustainability.

\section{References:}

European Commission, EC. (2008). EU Sustainable Development Strategy - EU SDS. Retrieved January 30, 2010, from http://ec.europa.eu/sustainable/welcome/idea_en.htm

Giesecke, M. (2002). Mythen der Buchkultur [Myths of book culture]. Frankfurt am Main: Suhrkamp.

Greenhow, C, Robelia B., \& Hughes J. E. (2009). Learning, teaching, and scholarship in a digital age. Web 2.0 and classroom research: What path should we take now? Educational Researcher, 38(4), 246-259. DOI: 10.3102/0013189X09336671.

Knipe, S., \& Edwards M. (2009). Teaching teachers to use blended learning. eLearn Magazine, 2009(11). Retrieved January 30, 2010, from http://www.elearnmag.org/subpage.c fm? section=articles\&article $=103-1$

Lambert, J., Kalyuga, S., \& Capan L. A. (2009). Student perceptions and cognitive load: What can they tell us about e-learning Web 2.0 course design? E-Learning, 6(2), 150163. Retrieved December 20, 2009, from http://dx.doi.org/10.2304/elea.2009.6.2.150

Mansell, R., \& Wehn U. (Eds.). (1998). Knowledge societies: Information technology for sustainable development. Oxford: Oxford University Press.

McCalla, G. (2004). The ecological approach to the design of e-learning environments: Purpose-based capture and use of information about learners. Journal of Interactive Media in Education, 7, 1-23. Retrieved January 30, 2010, from http://www-jime.open. ac.uk/2004/7

Mishra S., \& Sharma R. C. (2004). Interactive multimedia in education and training. Indira Gandhi National Open University.

Nystrom, C. (1973). Towards a science of media ecology: The formulation of integrated conceptual paradigms for the study of human communication systems. Unpublished doctoral thesis, New York University, New York, USA.

Peled, Z., Peled E., \& Gad A. (1994). An ecological approach for information technology. Intervention, evaluation and software adoption policies. In E. L. Baker \& H. F. O’Neil (Eds.), Technology assessment in education and training (pp. 35-61). New York: Routledge. 
Postman, N. (1970). The reformed English curriculum. In Eurich, A. C. (Ed.), High school 1980: The shape of the future in American secondary education (pp. 160-168). New York: Pitman.

Purg, P. (2006). An ecological approach to multimedia in teaching and training. Proceedings of Management International Conference Advancing Business and Management in Knowledge-Based Society, 23-25 November 2006 (pp. 1509-1514). Portorož, Slovenia: Faculty of Management.

Purg, P., \& Zakrajšek S. (2009). Technologies for sustainable teaching and learning: A case study from Slovenia on diminishing student workload and increasing motivation through ICT. Journal of Teacher Education for Sustainability, 11(2), 31-40. DOI: 10.2478/v10099-009-0038-x.

Sandbothe, M. (2004). Statement zur aktuellen Debatte über Mediologie, Medienökologie und Medienphilosophie [Statement of the current debate on mediology, media ecology and media philosophy]. Retrieved January 30, 2010, from http://www.sandbothe.net/3 9.html

United Nations Economic and Social Council, UNECE. (2009). Education for sustainable development. A strategy for the United Nations Decade 2005-2014. Retrieved January 30, 2010, from http://planipolis.iiep.unesco.org/upload/Denmark/Denmark_unsd_strat egy.pdf

United Nations Educational, Scientific and Cultural Organization, UNESCO. (2005). Guidelines and recommendations for reorienting teacher education to address sustainability. Retrieved January 30, 2010, from http://unesdoc.unesco.org/images/0014/0 01433/143370E.pdf

Tilbury, D. (2007). Learning based change for sustainability: Perspectives and pathways. In A. E. J. Wals (Ed.), Social learning (pp. 117-131). Wageningen: Wageningen academic publishers.

\section{Correspondence:}

Dr Peter Purg, Institute and Academy of Multimedia, Leskoškova 12, 1000 Ljubljana, Slovenia. Email: peter.purg@iam.si 